\title{
40. A HIGH-RESOLUTION 10-CENTIMETRE SURVEY OF THE GALACTIC CENTRE REGION AND SPECTRAL CHARACTERISTICS OF THE REGION
}

\author{
B. F. C. Cooper and R. M. Price \\ CSIRO Radiophysics Laboratory, Sydney
}

The region surrounding the galactic centre has been surveyed with the 210foot telescope of the Australian National Radio Astronomy Observatory at a wavelength of $10.0 \mathrm{~cm}$. At this wavelength the telescope beamwidth is 6.7 between half-intensity points.

The receiver was a crystal-mixer type which was temporarily used with the telescope prior to installation of a more sensitive parametric type. However, its sensitivity was adequate for observing the relatively high intensities found over most of the galactic centre region.

Figure 1 shows the isophotes of brightness temperature constructed from the observations, which extended over a region about 2 degrees square surrounding the galactic centre.

The brightness temperature scale was determined from observations of a number of calibrator sources, principally M87. For M87 a flux density of $110 \times 10^{-26}$ MKS units was adopted, based on intensity ratios to Cas A published by Sloanaker and Nichols (1960) and Heeschen and Meredith (1961) near $10 \mathrm{~cm}$ wavelength. The flux density of Cas A was taken as $1410 \times 10^{-26} \mathrm{MKS}$ units, adjusted to $10.0 \mathrm{~cm}$ from the absolute determination of $1340 \times 10^{-26}$ MKS units by Broten and Medd (1960) at $9.4 \mathrm{~cm}$. Other calibrator sources such as Taurus A, Orion A, and Hydra A, for which the adopted flux densities were respectively 750,400 , and $21 \times 10^{-26} \mathrm{MKS}$ units gave brightness temperature scales which were consistent within a few per cent of the scale deduced from M87. Appropriate allowance for beam broadening was made wherever present.

The features shown on Figure 1 are very similar to those originally discovered by Drake (1959) at $3 \cdot 75 \mathrm{~cm}$, except that the small peak at declination $-28^{\circ} 00^{\prime}$ lies outside the region covered by Drake.

The bright central source shows a slight elongation in declination, and, after subtracting contributions from underlying sources, we reduce equivalent gaussian widths of 3.'5 in right ascension and 4.0 in declination for this source. The equivalent linear dimensions would be about $12 \mathrm{pc}$ at an assumed distance of $10 \mathrm{kpc}$.

The spur source north of the central source shows some elongation across the plane while the nearby source at declination $-28^{\circ} 22^{\prime}$ shows elongation along the plane. The latter source, in conjunction with the minor source at declination $-29^{\circ} 25^{\prime}$, was supposed by Drake to be the projection of a ring of emission surrounding the galactic nucleus. At $10 \mathrm{~cm}$ these features are much less distinct than they are at $3.75 \mathrm{~cm}$. Underlying these small sources is a broad source, having a width of about $1: 5$ in longitude and 0.75 in latitude at half intensity. 
From comparisons of brightness temperatures on the outskirts of Figure 1 and those which would be expected from the $21-\mathrm{cm}$ continuum surveys of Westerhout (1958) and Mathewson, Healey, and Rome (1962), with appropriate adjustments for spectral slope, it is deduced that a zero correction of $+1 \cdot 5^{\circ} \mathrm{K}$ should be applied to the isophotes of Figure 1.



Fig. 1.-Galactic centre isophotes of brightness temperature at $3000 \mathrm{Mc} / \mathrm{s}$ in degrees Kelvin, observed with 6:7 beam. See text for zero correction to brightness temperature scale.

In calculating flux densities for the galactic centre region from these isophotes, an allowance must be made for foreground and background radiation from the galactic disk. Extrapolating $21-\mathrm{cm}$ survey data it is found that the disk contribution at $10 \mathrm{~cm}$ will be $3 \cdot 5^{\circ} \mathrm{K}$ along the ridge near the galactic centre, falling away to half value at latitudes $\sim \pm 1^{\circ}$.

Having made these adjustments the 10-cm flux densities for the region are as shown in Table 1. The flux for the central source is calculated as that contained in a gaussian pattern of peak brightness temperature $190^{\circ} \mathrm{K}$ and half-intensity widths $7: 6$ by $7: 9$, these being the measured widths of the beam as broadened by the source. The figure of $190^{\circ} \mathrm{K}$ is the difference between the observed peak brightness temperature of $210^{\circ} \mathrm{K}$ and an estimated $20^{\circ} \mathrm{K}$ contribution from the central extended source and the skirts of the spur source. The flux of the remaining components is obtained by integration of the isophotes with appropriate subtraction 
of underlying components. It is seen that the central extended source contributes by far the majority of the flux in this region.

TABLE 1

10-CM FLUX DENSITIES FOR GALACTIC CENTRE REgION

\begin{tabular}{l|rr|r|r}
\hline \multicolumn{1}{c|}{ Feature } & $\begin{array}{c}\text { Approximate } \\
\text { R.A. (1950) } \\
\text { h }\end{array}$ & $\mathrm{m}$ & $\begin{array}{c}\text { Approximate } \\
\text { Dec. (1950) }\end{array}$ & $\begin{array}{c}\text { Flux Density } \\
10^{-26} \mathrm{~W} / \mathrm{m}^{2}(\mathrm{c} / \mathrm{s})\end{array}$ \\
& & & & \\
Central source & 17 & $42 \cdot 5$ & $-28^{\circ} 58^{\prime}$ & 300 \\
Spur source & 17 & 43 & -2848 & 160 \\
North-east source 1 & 17 & 44 & -2822 & 55 \\
North-east source 2 & 17 & $45 \cdot 5$ & -2800 & 14 \\
South-west source & 17 & $41 \cdot 5$ & -2925 & 10 \\
Extended source & & & & 1520 \\
& & & & \\
\hline
\end{tabular}

The present 10-cm observations may be compared with those made by other observers at various wavelengths, with a view to determining the spectrum of the radiation. Table 2 shows some flux values for the central source reported by a number of observers. The value attributed to Drake at $3.75 \mathrm{~cm}$ is calculated from his isophotes for a peak brightness temperature of $25^{\circ} \mathrm{K}$ minus the contribution from the central extended source, which is estimated to be $2 \cdot 5^{\circ} \mathrm{K}$. A gaussian pattern of $7: 8$ diameter at half intensity is estimated for the beam as broadened by the source.

The scatter of the flux values in Table 2 makes it difficult to arrive at any definite conclusions about the spectrum of this source, but the balance of the evidence seems to point to a relatively flat spectrum.

TABLE 2

HIGH-RESOLUTION OBSERVATIONS OF THE GALACTIC CENTRAL SOURCE

\begin{tabular}{|c|c|c|c|}
\hline Observer & $\begin{array}{l}\text { Wavelength } \\
(\mathrm{cm})\end{array}$ & $\begin{array}{c}\text { Aerial } \\
\text { Resolution }\end{array}$ & $\begin{array}{c}\text { Flux Density } \\
10^{-26} \mathrm{~W} / \mathrm{m}^{2}(\mathrm{c} / \mathrm{s})\end{array}$ \\
\hline Cooper and Price & $10 \cdot 0$ & 6.7 pencil beam & 300 \\
\hline Little (1961) & $9 \cdot 0$ & $? \times 2.3$ fan beam & 286 \\
\hline Parijsky (1959) & $9 \cdot 4$ & $120^{\prime} \times 3^{\prime} .7$ fan beam & 150 \\
\hline & $3 \cdot 2$ & $40^{\prime} \times 11^{\prime} 1$ fan beam & 150 \\
\hline Drake (1959) & $3 \cdot 75$ & $6^{\prime}$ pencil beam & $260 *$ \\
\hline Howard, Rood, and Boyce (1962) & $3 \cdot 75$ & 6.2 pencil beam & 130 \\
\hline Biraud, Lequeux, and Le Roux (1960) & $21 \cdot 1$ & Interferometer & 280 \\
\hline Moffet and Maltby (1962) & 31 & Interferometer & 140 \\
\hline
\end{tabular}

* Calculated from isophotes.

In Table 3 the $10-\mathrm{cm}$ brightness temperatures of the main features in this region are compared with those shown on Drake's $3 \cdot 75-\mathrm{cm}$ isophotes. It is seen 
that the central source and the extended source appear to have a spectrum a little steeper than flat ("flat" being defined as having a $-2 \cdot 0$ index), while the other small features appear to have a somewhat inverted spectrum.

In the absence of more observations of these features at other wavelengths, these spectral indices must be regarded as very approximate. However, additional light is thrown on the nature of the emission in this region by Mills' (1958) 3.5 metre isophotes. At 3.5 metres a trough of absorption is noticed extending over about $1: 5$ of longitude near the galactic centre. This can be explained if the small central sources are thermal, and are seen (unresolved) in absorption against a nonthermal background provided by the central extended source. In support of this argument it may be pointed out that the absorption is asymmetrical towards positive galactic longitudes just as is the distribution of the small central sources.

TABLE 3

GALACTIC CENTRE REGION SPECTRAL INDICES

\begin{tabular}{l|c|c|c}
\hline \multicolumn{1}{c|}{ Feature } & $\begin{array}{c}T_{B} \text { at } 10 \mathrm{~cm}^{*} \\
\left(\begin{array}{c}\text { Present Survey) } \\
\left({ }^{\circ} \mathrm{K}\right)\end{array}\right.\end{array}$ & $\begin{array}{c}T_{B} \text { at 3.75 } \mathrm{cm}^{*} \\
(\text { Drake 1959) } \\
\left({ }^{\circ} \mathrm{K}\right)\end{array}$ & $\begin{array}{c}\text { Spectral } \\
\text { Index }\end{array}$ \\
\hline & & & \\
Central source & 192 & $22 \cdot 5$ & $-2 \cdot 2$ \\
Extended source & $15 \cdot 5$ & $2 \cdot 0$ & $-2 \cdot 1$ \\
Spur source & 42 & $8 \cdot 0$ & $-1 \cdot 7$ \\
North-east source 1 & 36 & $8 \cdot 0$ & $-1 \cdot 55$ \\
South-west source & 10 & $1 \cdot 5$ & $-1 \cdot 95$ \\
\hline
\end{tabular}

*After subtracting underlying sources.

For the extended source a nonthermal spectral index slightly steeper than $-2 \cdot 0$ would account for most of the $36,000^{\circ} \mathrm{K}$ brightness temperature observed near the galactic centre at $3 \cdot 5$ metres, after allowance for the contribution of the disk radiation.

Alternatively it is possible that both thermal and nonthermal emission are present in the extended source. For various spectral indices of the nonthermal component, it is possible to compute suitable proportions of thermal and nonthermal emission which will account for both the microwave and metre-wave intensities of the extended source.

For instance, the 10 -cm brightness temperature of $15.5^{\circ} \mathrm{K}$ given in Table 3 may be considered to be composed of a homogeneous mixture of about $75 \%$ thermal emission and $25 \%$ nonthermal emission with a spectral index of $-2 \cdot 7$. A calculation following Westerhout (1958), allowing for the substantial optical depth of the above thermal component at 3.5 metres and also the emission and absorption in the disk component, will then give to a good approximation the observed $3 \cdot 5$-metre brightness temperature in the region of the galactic centre.

\section{Polarization}

No systematic search of the region for linear polarization has been made as yet. However, the central source appears to be less than $0.5 \%$ polarized at $10 \mathrm{~cm}$ 
while the source at R.A. $17^{\mathrm{h}} 44^{\mathrm{m}}$, declination $-28^{\circ} 22^{\prime}$ appears to be less than $1 \cdot 5 \%$ polarized. One would expect strong depolarization effects over most of the galactic centre region.

\title{
Position of the Galactic Centre
}

For the peak of the central source of Figure 1 we find the $1950 \cdot 0$ coordinates:

Right ascension $17 \mathrm{~h} 42^{\mathrm{m}} 30 \mathrm{~s} 0 \pm 2^{\mathrm{s}}$

Declination $\quad-28^{\circ} 58: 7 \pm 0 ! 5$.

This position is a pproximately 4.0 east and 3.7 south of the new IAU coordinates of the galactic centre. A small error may be noted in the positioning of the coordinate grid of Figure 1, which was plotted before the final coordinates were calculated.

\section{Acknowledgments}

We thank Miss Janice A. Milton for assistance in preparing the 10 -cm isophotes, and Mr. T. E. Cousins for setting up the receiver. One of us (R.M.P.) held a Fulbright Fellowship during the course of this work.

\section{References}

Biraud, F., Lequeux, J., and Le Roux, E. (1960).-Observatory 80: 116-18.

Broten, N. W., and Medd, W. J. (1960).-Ap. J. 132 : 279-85.

Drake, F. D. (1959).-N.R.A.O. Annual Report No. 1: 2.

Heeschen, D. S., and Meredith, B. L. (1961).-Publ. N.R.A.O. 1 : 121-8.

Howard, W. E., III, Rood, H. J., and Boyce, P. B. (1962).-Ap. J. 136 : 133-7.

Littue, A. G. (1961).-Stanford Radio Astronomy Inst. Publ. No. 16.

Mathewson, D. S., Healey, J. R., and Rome, J. M. (1962).-Aust. J. Phys. 15 : 354-77.

Mirls, B. Y., Litthe, A. G., Sheridan, K. V., and Slee, O. B. (1958).-Proc. I.R.E. 46: 67-84. Moffet, A. T., and Maltby, P. (1962).-Ap. J. Suppl. $7:$ 93-163.

PariJsky, Y. N. (1959).-Doklady Akad. Nauk SSSR 129: 1261-3; Soviet Phys. Doklady 4 : $1172-4$. Sloanaker, R. M., and Nichols, J. H. (1960).-U.S. Naval Research Laboratory Rep. 5485.

Westerhout, G. (1958).-B.A.N. 14: 215-60.

\section{Discussion}

Davies: Your derivation of the spectral index of the source near the galactic centre using 10 and $3.75 \mathrm{~cm}$ is important. How was the $3.75 \mathrm{~cm}$ brightness temperature calibration of Drake's isophote map derived?

Cooper: I do not know how Dr. Drake calibrated his brightness temperature scale. His map was stated to show isophotes of brightness temperature directly in degrees $\mathrm{K}$. Lequeux says he believes the scale to be in $T_{a}$ rather than in $T_{b}$, thus causing the derived $T_{b}$ to take on a higher value.

\section{THE STRUCTURE OF THE GALACTIC NUCLEUS}

\author{
Y. N. PariJsky \\ Pulkovo Observatory
}

The problem of the nuclei of galaxies is really one of the most important problems on the way to our understanding of numerous very strange phenomena which we observe in our own Galaxy as well as in many others, especially in radio galaxies. 